\title{
V. Die Günzburger Öffentlichkeit und der Fall Mengele 1945-1959
}

„Fünf schöne Jahre verlebte Mengele seelenruhig in Günzburg; er fuhr oft nach München und in andere Städte. Niemand behelligte ihn. Erst [...] 1951 floh er über die Route Reschenpaß-Meran nach Italien, von dort nach Spanien und später weiter

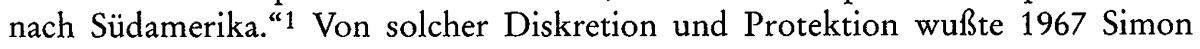
Wiesenthal zu berichten - nichts davon stimmte. Doch auch wenn die Günzburger Josef Mengele nach dem Krieg nachweislich nicht jahrelang versteckt hielten oder er gar offen dort leben konnte ${ }^{2}$, bleibt dennoch die Frage, ob und wie viel die Günzburger von den Verbrechen Josefs, des Sohnes des angesehenen und bewunderten Fabrikanten Karl Mengele, wußten und wie sie mit diesem Wissen umgegangen sind.

Am 3. Mai 1945 zählten die Alliierten in einer Rundfunkmeldung die Verbrechen auf, die Josef Mengele zur Last gelegt wurden ${ }^{3}$ : $\mathrm{He}$ was one of the Chief selectors in the camp. [...] He also used [twins and liliputians] for his famous experiments." 4 $\mathrm{Ob}$ und in welchem Ausmaß die Günzburger zu diesem Zeitpunkt diese Vorwürfe zur Kenntnis genommen haben, ist nicht bekannt. Als gesichert kann jedoch gelten, daß die amerikanischen Besatzungstruppen während ihres gesamten Aufenthalts nicht außergewöhnlich intensiv nach Mengele gesucht haben. Der Besuch eines amerikanischen Militärpolizisten und seines Übersetzers bei Irene Mengele in Autenried (einem kleinen Ort in der Nähe von Günzburg, wo Irene mit ihrem kleinen Sohn Rolf mittlerweile lebte) am 11. Juni 1945 war „the only U.S. effort to locate Josef Mengele in occupied Germany" "5. Dabei scheint es sich aber nicht um die Fahndung nach einem gesuchten Kriegsverbrecher gehandelt zu haben. In diesen Tagen wurde in der Gegend um die Stadt eine ganze Reihe von NS-Funktionären verhaftet, die unter die Kriterien des automatischen Arrests fielen. ${ }^{6}$ „Information available locally as well as information gleaned from an interrogation of Karl Mengele, Sr. would likely have given sufficient cause for Josef Mengele to be picked

1 Wiesenthal, Mörder, S. 206.

2 Auch das OSI, das das Verhalten der Amerikaner im Fall Mengele untersuchte, widmete dieser Frage eine ausführliche Betrachtung: "This claim implies at least ignorance and at worst acquiescence or complicity on the part of the U.S. authorities stationed there", and therefore "considerable resources were devoted to determining the facts behind the allegation". Vgl. In the Matter of Josef Mengele 1, S. 53-62, Zitate S. 53 und 58.

3 Vgl. Tagcbucheintrag Irene Mengeles, nach: Posner/Ware, Mengele, S. 91.

4 In the Matter of Josef Mengele 1, S. 99. Dort wird ein Radioskript des Psychological Warfare Department der 9. US-Armce, 28. 4. 1945, zitiert, das nicht unbedingt mit der Radiosendung identisch sein muß, die Irene Mengele fünf Tage später in Günzburg gehört hat.

5 Ebenda, S. 94.

6 So etwa am 11.6. 1945, also dem gleichen Tag, an dem die Militärstreife auch Irene Mengele befragte, außerdem am 4. und am 18.6. Vgl, ebenda. 
up and his wife questioned."7 Die Amerikaner suchten Mengele also nicht wegen seiner Verbrechen in Auschwitz, sondern allein wegen seines Ranges als Hauptsturmführer in der Waffen-SS. ${ }^{8}$

Dies bestätigten auch die wenigen Angehörigen der amerikanischen Stadtkommandantur, die sich bei der Befragung durch das OSI an den Namen Mengele erinnern konnten, darunter auch ein Emigrant jüdischer Herkunft, der nach eigenen Angaben „particularly sensitive to war crime matters" 9 war. Das erklärt, warum sich die Amerikaner mit der Erklärung Irene Mengeles zufrieden gaben, sie habe keine Nachricht von ihrem Mann, er sei im Osten vermißt und wahrscheinlich tot. Im Juni 1945 mag sie dies möglicherweise tatsächlich geglaubt haben. Als sie spätestens Mitte August erfuhr, daß ihr Mann lebte, gab sie dennoch weiterhin die Soldatenwitwe - und zwar nicht nur gegenüber den Amerikanern, sondern auch gegenüber ihren Freunden und Bekannten und erst recht gegenüber der Günzburger Öffentlichkeit, immer in der Angst, jemand könnte die Amerikaner informieren. In den Briefen, die sie in dieser Zeit schrieb, sprach sie von ihrer "feste[n] Annahme, $\mathrm{da} ß$ ich meinen Mann nie mehr lebend sehen werde", sie trug schwarze Kleidung und bat den Pfarrer, für das Seelenheil ihres Mannes zu beten. ${ }^{10}$ Der Rest der Familie Mengele dürfte sie in ihrer Rolle als trauernde Witwe nach Kräften unterstützt haben.

Das Mengele'sche Theaterstück war ein voller Erfolg. Dies zeigte sich - sozusagen auf höchster Ebene - 1948, als sich die Fehlinformation bis zu Telford Taylor, dem amerikanischen Chefankläger vor dem Internationalen Militärtribunal (IMT) in Nürnberg, herumgesprochen hatte. Der Name Josef Mengele war mittlerweile in mehreren Nachkriegsprozessen, so etwa noch 1945 im Bergen-Belsen-Prozeß oder 1946 vor dem IMT, gefallen. ${ }^{11}$ Dennoch suchten die Amerikaner auch weiterhin nicht nach Mengele. Den Grund hierfür schrieb Taylor Anfang 1948 an die Schriftstellerin Gisella Perl, die selbst ein Opfer Mengeles war und sich nach einer falschen Verhaftungsmeldung 12 als Zeugin zur Verfügung stellen wollte: „With reference to your letter dated 8 December 1947 [...] we wish to advise our records show Dr. Mengerle [sic!] is dead as of October 1946."13

Die Amerikaner in Günzburg interessierten sich also nicht besonders für Josef Mengele, er hatte keinen besonderen Status oder gar Priorität; entsprechend sahen sie auch keinen Grund, die Auskunft, er sei tot, zu hinterfragen. Auch die Günzburger dürften das dank des schauspielerischen Talents von Irene Mengele zunächst geglaubt haben - soweit sie sich denn überhaupt dafür interessierten. Sie hatten, wie die Amerikaner, zunächst andere Sorgen in einer Stadt, deren Bevölkerung durch Evakuierte, Flüchtlinge und Vertriebene im Vergleich zu 1939 um

7 Ebenda, S. $94 \mathrm{f}$.

8 Vgl. ebenda, S. 90-101.

9 Ebenda, S. 60.

$10 \mathrm{Vgl}$. Posner/Ware, Mengele, S. 108, Zitat ebenda.

11 Vgl. die Prozeßprotokolle: Trial of Josef Kramer, S $130 \mathrm{f}$. und $250 \mathrm{f}$., sowie Prozeß gegen die Hauptkriegsverbrecher 11, S. 448.

12 Die Meldung stand im Zusammenhang mit dem „Gorby-Memorandum“, vgl. In the Matter of Josef Mengele 1, S. 70-90, und Bd. 2, S. 86-90.

13 Brief Telford Taylors an Gisella Perl betr. Information regarding Dr. Mengerle [sic!], 19. 1. 1948, in: In the Matter of Josef Mengele 2, S. 91. 
rund 50 Prozent zugenommen hatte. ${ }^{14}$ Dazu kam, daß die Informationen über die Shoa in Deutschland zwar schnell bekannt und in nuce auch als wahr akzeptiert wurden, die Bevölkerung aber gleichzeitig vieles von dem, was an Schrecklichem aus den Lagern berichtet wurde, für überzogene alliierte Greuelpropaganda hielt.. ${ }^{15}$ Sowohl die „alten“ als auch die „neuen“ Günzburger dürften dem Schicksal des Josef Mengele angesichts der eigenen existentiellen Sorgen kaum Aufmerksamkeit geschenkt haben - ebenso wenig wie im Deutschland dieser Zeit die Opfer der rassischen oder politischen Verfolgung mit besonderer Anteilnahme rechnen konnten. 16

Die sich langsam bessernde wirtschaftliche Lage änderte das verbreitete Desinteresse an der jüngsten Vergangenheit zunächst nicht. Die Entnazifizierung wurde beendet, Belastete auf breiter Basis rehabilitiert. ${ }^{17}$ Wenn die Deutschen in den 1950er Jahren überhaupt zurückschauten, dann sahen sie sich selbst als Opfer - obwohl in der publizistischen Öffentlichkeit sehr wohl über die NS-Vergangenheit und die Schuld der Deutschen gestritten wurde und immer wieder Skandale die junge, noch nicht gefestigte Republik erschütterten. ${ }^{18}$ Aleida Assmann hat in diesem Zusammenhang von „zwei Kulturen“ gesprochen, die im Nachkriegsdeutschland und der frühen Bundesrepublik existierten: „Eine vom Paradigma der Schuld geprägte Öffentlichkeitskultur und eine vom Paradigma der Scham geprägte Kultur des Schweigens"19 im privaten Bereich. Es herrschte, so Norbert Frei, eine „Atmosphäre des bereitwilligen Beschweigens individueller NS-Vergangenheit“20. Der Ruf nach einem Schlußstrich wurde lauter, und die Mehrheit, die sich selbst als Opfer sah, wollte von den Verbrechen, die Deutsche im Namen des Nationalsozialismus begangen hatten, nichts mehr hören und wissen. ${ }^{21}$

Günzburg bildete hier keine Ausnahme; das belegt nicht zuletzt das Schicksal Richard Böcks, der ebenfalls aus Günzburg stammte und als Kraftfahrer in Auschwitz gewesen war. Böck sprach öffentlich über seine Erlebnisse und wurde daraufhin in seiner Heimatstadt geschnitten. ${ }^{22}$ Der Schluß, daß eine solche Stadt dann auch Josef

14 Von 6986 am 17. 5. 1939 auf 10425 am 1. 7. 1948. Von den Neubürgern waren 1823 Flüchtlinge, 473 Evakuierte und 147 Ausländer. Vgl. Sallinger, Integration, S. 290.

15 Schildt, Umgang, S. 28.

16 Vgl. ebenda, S. 30.

$17 \mathrm{Zu}$ nennen wären hier neben der Liquidation der Entnazifizierung die Straffreiheitsgesetze von 1949 und 1954 und die „131er"-Regelung. Vgl. Frei, Vergangenheitspolitik, S. 25-131.

$18 \mathrm{Zu}$ denken wäre hier etwa an den Remer-Prozeß, das Verbot der neonationalsozialistischen SRP, die Wiederbewaffnungsdebatte, die Kriegsverbrecherfrage oder die Bewertung des 20. Juli sowie an zahlreiche Skandale, die sich an den Biographien einzelner Personen entzündeten (Hans Globke, Theodor Oberländer, Veit Harlan, Werner Heyde/Sawade). Vgl. Reichel, Vergangenheitsbewältigung, S. 19 und 138-152; Steinbach, S. 38-40, und Frei, Vergangenheitspolitik.

19 Assmann/Frevert, Geschichtsvergessenheit, S. 111.

20 Frei, Vergangenheitspolitik, S. 405.

21 Vgl. Schildt, Umgang, S. 34-36.

22 Viele derer, die über die NS-Verbrechen sprechen wollten, teilten dieses Schicksal: Sie wurden für die Mehrheit der Deutschen zu Nestbeschmutzern. Hermann Langbein schildert in seinem Buch „Menschen in Auschwitz" mehrere solcher Fälle. Vgl. Langbein, Menschen, insb. S. 481 und 573 f.; vgl. außerdem: „Wenn er kam, waren wir vor Angst wie gelähmt“, in: GZ, 6. 11. 1984 . 
Mengele versteckt, protegiert oder gar bewundert hätte, geht allerdings fehl. Die NS-Verbrechen waren während der 1950er Jahre schlicht ein Thema, mit dem die Menschen nicht nur in Günzburg nichts zu tun haben wollten, das „beschwiegen“ wurde. Sie interessierten sich nicht dafür, und damit auch nicht für die Verbrechen Josef Mengeles in Auschwitz. Das bedeutete jedoch nicht, daß sich so mancher Günzburger nicht hinter vorgehaltener Hand gefragt hätte, was denn nun aus dem Beppo, dem ältesten Sohn des angesehenen Fabrikbesitzers, geworden war. In der Kleinstadt machten Gerüchte schnell die Runde. So manche Information scheint ihren Ursprung auch direkt bei der Familie Mengele gehabt $\mathrm{zu}$ haben ${ }^{23}$, die bald, genau wie Josef Mengele in Argentinien ${ }^{24}$, keine Veranlassung mehr sah zu übertrieben strenger Geheimhaltung. Dies lag aber keineswegs daran, daß sie an eine besondere Verschwiegenheit ihrer Mitbürger geglaubt oder es gar heimliches Komplizentum gegeben hätte; vielmehr schien sich Mitte der 1950er Jahre in Deutschland schlicht niemand mehr - und das schließt die Justiz ausdrücklich ein - für Josef Mengeles Verbrechen zu interessieren.

Seit 1953 war die Verfolgung von NS-Verbrechern durch deutsche Gerichte fast völlig zum Erliegen gekommen ${ }^{25}$, die NS-Belasteten waren zum allergrößten Teil in den neuen Staat integriert worden und gerade bei den gesellschaftlichen Eliten war eine weitgehende personelle Kontinuität zum Dritten Reich festzustellen. ${ }^{26}$ Gegen Ende der 1950er Jahre hatte sich die Bundesrepublik als stabiler Staat erwiesen. Die Deutschen hatten sich in ihrem neuen Wohlstand und in ihrer neuen politischen Stellung in der Welt eingerichtet und eine neue Generation derer, die bei Kriegsende noch Kinder gewesen waren, begann, sich zu artikulieren. Die „Kultur des Schweigens" wurde erstmals nicht mehr nur durch einzelne Mahner hinterfragt. ${ }^{27}$

Am Beginn dieser Entwicklung stand vor allem ein spektakuläres Ereignis: Der sogenannte Ulmer Einsatzgruppenprozeß ${ }^{28}$ von 1958 , der die „gravierenden Unter-

23 Daß Martha Mengele mit ihrem Sohn Karl-Heinz plötzlich nach Südamerika ging, dürfte in einer Kleinstadt wie Günzburg kaum verborgen geblieben sein; daraus konnte man natürlich Schlüsse ziehen, und Karl Mengele selbst scheint sich dahingehend gegenüber seiner Haushälterin geäußert zu haben. Vgl. S. 121.

$24 \mathrm{Vgl}$. S. $52 \mathrm{f}$.

25 Nach einem Höchststand von 1819 Verfahren im Jahr 1948 hatte es 1950 noch 809 und 1953 noch 123 Verfahren gegeben; danach schwankte die Zahl bis 1961 zwischen etwa 20 und 40, der Tiefstand war 1959 mit 15 Verfahren erreicht. Vgl. Garbe, Abkehr, S. 703. Zur Geschichte der NS-Prozesse vgl. Rückerl, NS-Verbrechen und zu ihren gesellschaftlichen Auswirkungen Steinbach, Gewaltverbrechen.

26 Deshalb war schnell die Rede von einer „Renazifizierung“; dieser Begriff sorgte in späteren Jahren für Mißverständnisse: „Denn [... ] zum einen hatte ein Großteil der begünstigten Personen bereits vor 1933 wie im NS-Regime funktioniert, zum zweiten bildete deren strenge Abgrenzung von rechtsextremer Aktivität eine Bedingung für die soziale Integration als Chance einer zweiten Sozialisation." Schildt, Umgang, S. 38. Vgl. zum Thema der Integration der NS-Eliten in die bundesrepublikanische Gesellschaft Herbert, NS-Eliten.

27 Vgl. Schildt, Umgang, S. 45 und 49; Garbe, Abkehr, S. 707 ff., und Miquel, NS-Prozesse, S. 112.

28 Ein ehemaliger SS-Oberführer (ein Rang ohne Wehrmachtspendant zwischen Oberst und Generalmajor), der Polizeidirektor von Memel gewesen und dort 1941 an Massenerschießungen von Juden beteiligt gewesen war, war bereits 1956 verhaftet worden und wurde vom Schwurgericht Ulm 1958 neben weiteren Gestapomitgliedern zu einer Freiheitsstrafe von 12 Jahren verurteilt. Nach dem Krieg hatte der Angeklagte unter falschem Namen ein Flücht- 
lassungen und skandalösen Fehlleistungen " 29 bei der Verfolgung von NS-Verbrechern durch die Ermittlungsbehörden offenlegte. ${ }^{30}$ Bereits 1955 waren alle Straftaten, die mit bis zu zehn Jahren Gefängnis belegt waren, verjährt - darunter fiel z.B. auch die schwere Körperverletzung. 1960 nun drohten auch Straftaten wie Totschlag und Körperverletzung bzw. Freiheitsberaubung mit Todesfolge zu verjähren. Nachdem im Ulmer Einsatzgruppenprozeß offenbar geworden war, daß viele der im Osten begangenen NS-Verbrechen noch nicht gesühnt, ja manche Täter im Entnazifizierungsverfahren sogar als "nicht betroffen" eingestuft worden waren, entschlossen sich die Justizminister der Länder im Oktober 1958, die Zentrale Stelle der Landesjustizverwaltungen zur Aufklärung von nationalsozialistischen Gewaltverbrechen ${ }^{31}$ ins Leben zu rufen. Diese nahm am 1. Dezember in Ludwigsburg ihre Arbeit auf und hatte den Auftrag, alle verfügbaren Unterlagen über NS-Verbrechen zu sammeln, Tatkomplexe herauszuarbeiten und die Täter zu ermitteln. Dadurch sollte ermöglicht werden, noch vor Ablauf der anstehenden Verjährungsfristen möglichst viele Verfahren einzuleiten.

Gleichzeitig erschütterten verschiedene Skandale die Republik, die besonders die Frage aufwarfen, wie es die Deutschen 15 Jahre nach Ende des Dritten Reiches mit dem Antisemitismus hielten. Die Medien berichteten das ganze Jahr 1959 hindurch von antisemitischen Vorfällen: In Düsseldorf beschmierten Unbekannte die Synagoge mit Hakenkreuzen, in Freiburg wurde ein jüdischer Friedhof geschändet. In Hamburg wurde ein Kaufmann wegen antisemitischer Äußerungen zu einer Haftstrafe verurteilt, und in Frankfurt mußten sich die sogenannten Köpperner Antisemiten, die ein jüdisches Ehepaar beleidigt, bedroht und verfolgt hatten, vor Gericht verantworten. Bereits in den Jahren zuvor hatte es ähnliche Fälle gegeben, und es war nicht mehr zu übersehen, daß in der Gesellschaft antisemitisches Potential vorhanden war. ${ }^{32}$ Der Publizist Georg von Studnitz prophezeite, das Jahr 1959 werde in die Geschichte eingehen als das Jahr, in dem „das Problem des Antisemitismus“33 erstmals seit 1933 wieder in der breiten Öffentlichkeit debattiert worden sei. Doch das Jahr war noch nicht zu Ende: Am Heiligabend wurde die Kölner Synagoge durch Hakenkreuzschmierereien geschändet, und die Empörung war diesmal nicht zuletzt durch den besonderen Tatzeitpunkt - größer als jemals zuvor, sie war „allgemein und parteiübergreifend, [...] international und bemerkenswert nachhaltig“34. Gleichzeitig geriet die Regierung Adenauer ob ihres belasteten Personals immer stärker unter Druck, nicht zuletzt durch die Propaganda der SED-Führung in Ost-Berlin. ${ }^{35}$

lingslager geleitet und war, als man ihn erkannt hatte, entlassen worden. Später klagte er auf Wiedereinstellung in den Staatsdienst; im Verlauf dieses Verfahrens wurde er als Polizeichef von Memel identifiziert. Vgl. Reichel, Vergangenheitsbewältigung, S. 145, und Steinbach, Gewaltverbrechen, S. $46 \mathrm{ff}$.

29 Garbe, Abkehr, S. 707.

$30 \mathrm{Vgl}$. zu den Auswirkungen des Prozesses auch: Miquel, NS-Prozesse, S. $100 \mathrm{f}$.

$31 \mathrm{Zu}$ Gründung und Tätigkeit der Zentralen Stelle vgl. Dreßen, Zentrale Stelle, und Steinbach, Gewaltverbrechen, S. $48 \mathrm{ff}$.

32 Reichel, Vergangenheitspolitik, S. 147.

33 Zit. nach Reichel, Vergangenheitspolitik, S. 147.

$34 \mathrm{Vgl}$. Reichel, Vergangenheitspolitik, S. $147 \mathrm{f}$.

35 Vgl. Schildt, Umgang, S. 50-52. 
Die Deutschen sahen sich erneut mit ihrer bereits bewältigt geglaubten Vergangenheit konfrontiert (das Schlagwort der „Vergangenheitsbewältigung“ stammt aus der Mitte der 1950er Jahre ${ }^{36}$ ) und mußten feststellen, „daß die Nazis Deutsche, und daß sehr viele Deutsche Nazis gewesen waren "37. Politische Bildung und Wissenschaft ${ }^{38}$ nahmen sich nun verstärkt des Themas an, und auch die Presse, Film, Rundfunk und Fernsehen beteiligten sich an dieser "zeithistorischen und geschichtspolitischen Offensive“39. Im September 1959 hatte der Film „Rosen für den Staatsanwalt" Premiere, 1960 zeigte der WDR-Mehrteiler „Am Grünen Strand der Spree" erstmals - fiktionale - Bilder der Judenvernichtung in Polen und von Oktober 1960 bis Mai 1961 sahen rund 15 Mio. Fernsehzuschauer jeden Freitagabend zur besten Sendezeit die mehrteilige Dokumentation „Das Dritte Reich“. Rund 180 Sendungen haben ARD und ZDF in dieser Zeit über den Nationalsozialismus ausgestrahlt. ${ }^{40}$ Besonders bedeutsam jedoch wurde ein schmales Buch, dessen 1955 erstmals publizierte Taschenbuchausgabe nach 40000 verkauften Büchern im ersten Jahr drei Jahre später bereits eine Auflage von rund 750000 erreicht hatte. ${ }^{41}$ Es war dieses Einzelschicksal eines kleinen jüdischen Mädchens, das sich mit seiner Familie in Amsterdam versteckt gehalten und zwischen 1942 und 1944 Tagebuch geführt hatte, das die Menschen bewegte: Das Tagebuch der Anne Frank. ${ }^{42}$

Das große Interesse, auf das die Aufzeichnungen Anne Franks stießen, sollte für Josef Mengele Folgen haben. Der Journalist Ernst Schnabel verfolgte die Spur des Kindes, um so viel wie möglich über ihr Leben und Sterben herauszufinden. Sein im März 1958 erschienenes Buch „Anne Frank. Spur eines Kindes“ 43 wurde viel beachtet und eine ganze Reihe von Zeitungen druckte es in Fortsetzungen. Unter anderem beschrieb Schnabel die mörderischen Bedingungen in Auschwitz und beklagte, daß von den SS-Leuten viele einfach verschwinden konnten: „Keiner weiß zum Beispiel, wo Dr. Mengele ist, ob er umkam oder ob er heute noch irgendwo lebt. Dr. Mengele war der Arzt, der bei den Selektionen unter dem Scheinwerfer stand und [...] nach rechts schickte oder nach links, je nachdem..."44

Eine der Zeitungen, die Auszüge aus Schnabels Buch druckten, waren die Ulmer Nachrichten, und der oben zitierte Absatz war in der Ausgabe vom 1. Juli $1958 \mathrm{zu}$ lesen. Wenige Tage später erreichte die Redaktion ein anonymer Brief einer ,junge[n] Leserin“, die schrieb, daß „scheinbar [...] doch einige Leute“ wüßten, wo sich Mengele aufhalte: „Sonst hätte nicht der alte Herr Mengele in Günzburg

36 Auf den schwierigen Versuch, diesen Begriff zu definieren, soll hier verzichtet werden; ohnehin steht seine geradezu inflationäre Nutzung im Gegensatz zu seiner begrifflichen Schärfe. Statt dessen sei hier verwiesen auf: Dudek, Vergangenheitsbewältigung, S. 44-53, sowie die Anmerkungen Ulrich Brochhagens in: Brochhagen, Nürnberg, S. $11 \mathrm{f}$. Dennoch kommt auch diese Arbeit um die Verwendung dieses Begriffes nicht herum.

37 Vgl. Reichel, Vergangenheitspolitk, S. 147.

38 Vgl. zur Bedeutung des zunehmenden Interesses der Geschichtswissenschaft an der Thematik des Dritten Reiches: Schildt, Umgang, S. $45 \mathrm{f}$.

39 Ebenda, S. 149.

40 Vgl. ebenda, S. $149 f$.

41 Vgl. Mit Anne Frank begann die Erinnerung, in: Die Welt, 7. 5. 2002. Die Erstausgabe von 1950 hatte sich dagegen so gut wie gar nicht verkauft.

42 Frank, Tagebuch.

43 Schnabel, Anne Frank.

44 Ebenda, S. 138. 
[Randbemerkung: bei Ulm] seiner ehemaligen Hausgehilfin Frau Angela K. in Steinheim/Neu-Ulm erzählt, daß sein Sohn, der Arzt bei der SS war, in Südamerika unter einem anderen Namen eine Praxis ausübt. Und weil er so Heimweh habe, hat Herr Mengele die Witwe seines anderen Sohnes nach drüben geschickt." 45 Die Redaktion leitete den Brief weiter an Schnabel, der ihn kurz darauf seinerseits an die Staatsanwaltschaft in Ulm sandte. Der dortige Oberstaatsanwalt Schüle interpretierte Schnabels Begleitbrief als Strafanzeige und gab die Schriftstücke wenige Tage später zuständigkeitshalber an die Staatsanwaltschaft Memmingen weiter, die für Günzburg örtlich zuständig war. ${ }^{46}$ Von nun an gab es einen Fall Josef Mengele. ${ }^{47}$

Noch im gleichen Monat wandte sich die Staatsanwaltschaft Memmingen an die „Kriminals-Außenstelle [sic!] Günzburg“ mit dem „Ersuchen, die Personalien des Beschuldigten und, wenn möglich, seinen derzeitigen Wohnsitz [...] festzustellen" 48. Der Brief schloß mit der Anweisung, "die Ermittlungen so durchzuführen, daß sie den Angehörigen nicht zur Kenntnis kommen"49 - eine Aufforderung, die von zumindest einem Günzburger Beamten ignoriert wurde. Rolf Mengele hat die Existenz eines Informanten bei der Günzburger Polizei später bestätigt. ${ }^{50}$ Kaum hatten die Ermittlungen begonnen, waren die Familie in Günzburg und Josef Mengele in Buenos Aires gewarnt.

Die Günzburger Polizei wandte sich an die Stadtverwaltung und erhielt, wie explizit festgehalten wurde, „vertrauliche Auskunft von Oberbürgermeister Dr. Josef Seitz persönlich“51. „Zusammenfassend“ konnten die Günzburger Beamten Ende August nach Memmingen melden, daß „der letzte polizeiliche Meldeort des Dr. Josef Mengele von 1943 bis 1944 der Wohnsitz seiner Familie in Freiburg/Breisgau, Sonnenhalde Nr. 87 war, und daß sich dieser zuletzt im Jahre 1954 aus der Hauptstadt von Argentinien, Buenos Aires, Sarmiento 1875 Olivos, meldete“52. In Freiburg hatte Mengele im November 1944 seinen letzten Heimaturlaub bei seiner Frau Irene verbracht, die Adresse in Argentinien dürfte den Günzburgern aus den Vorgängen um Josef Mengeles Ehescheidung im Jahr 1954 bekannt gewesen sein. Jedenfalls stand nun fest, daß die Staatsanwälte in Memmingen für den Fall nicht zuständig waren. Noch Ende August gaben sie den Fall ab an ihre Kollegen in

45 Sta F/M, Az 4 Js 340/68, Ermittlungsakten, Bd. I, Bl. 7, Anonymer Leserbrief, 8. 7. 1958.

46 Ebenda, Bl. 3-5, Schreiben Schnabels an die Staatsanwaltschaft Ulm, 3. 8. 1958, weitergeleitet an die Staatsanwaltschaft Memmingen am 12. 8. 1958. Vgl. hierzu auch Völklein, Mengele, S. $251 \mathrm{f}$.

47 Die von Posner und Ware geschilderte Version, wonach Bemühungen Hermann Langbeins, des Generalsekretärs des Wiener Auschwitz-Komitees, zur Einleitung des Strafverfahrens geführt hätten, können dagegen in den Akten nicht nachvollzogen werden und sind deswegen - trotz des verdienstvollen Engagements Langbeins - wohl falsch. Vgl. Posner/Ware, Mengele, S. 151-154.

48 Sta F/M, Az 4 Js 340/68, Erm.A., Bd. I, Bl. 11, Schreiben der Staatsanwaltschaft Memmingen an die Landpolizei Günzburg, August 1958.

49 Ebenda.

50 Vgl. Posner/Ware, Mengele, S. 378. Vgl. außerdem Völklein, Mengele, S. $252 \mathrm{f}$.

51 Sta F/M, Az 4 Js 340/68, Erm.A., Bd. I, Bl. 15, Protokoll der Bayerischen Landpolizei, Kriminalaußenstelle Günzburg, 23.8.58.

52 Ebenda, Bl. 33-36, Bericht der Polizei Günzburg an die Staatsanwaltschaft Memmingen über die bisherigen Ermittlungsergebnisse, 25. 8. 1958. 
Freiburg 53 , und der dortige Oberstaatsanwalt Schabinger Freiherr von Schowingen entwickelte eine für die - bisher an der Verfolgung von NS-Verbrechern wenig interessierte - deutsche Justiz durchaus bemerkenswerte Initiative, die rund ein halbes Jahr später, am 25. Februar 1959, zum ersten Haftbefehl gegen Josef Mengele führte. ${ }^{54}$

53 Vgl. ebenda, Bl. 37, Schreiben der Staatsanwaltschaft Memmingen an die Staatsanwaltschaft Freiburg i. Br. betr. Übernahme zuständigkeitshalber, 30. 8. 1958.

54 Vgl. ebenda, Bl. 287, Haftbefehl d. Amtsgerichts Freiburg, 25. 2. 1959. 